\title{
Finite Similitude in Metal Forming
}

\author{
Keith Davey ${ }^{1 \mathrm{a}}$, Rooholamin Darvizeh ${ }^{1}$ and Anees Al-Tamimi ${ }^{1}$ \\ ${ }^{1}$ School of MACE, The University of Manchester, Manchester, UK
}

\begin{abstract}
Scaled experimentation founded on dimensional analysis has a long history but has to-date achieved little success in complex metal-forming applications. This is particularly true for thermo-mechanical processes, where scaled experimentation is recognised to provide little insight. It is shown in this paper that current similarity approaches in continuum physics can be overly prescriptive. The concept of finite similitude is introduced, which is shown to be better suited to finite-element analysis for the design of scaled experiments.
\end{abstract}

\section{Introduction}

Scaling and in particular dimensional analysis plays an important role in experimental continuum physics. The most well-known and important theorem in modern dimensional analysis is the Buckingham Pi theorem [1], which quantifies the number of dimensionless quantities. If it transpires that the number and magnitude of the dimensionless quantities of the full-scale model and the scaled model coincide, then the two models are denoted similar and accordingly produce corresponding physics. But in reality, metal forming processes are complex and it is not generally possible to find an exact similar model. Although it is evident that dimensional analysis has an important part to play in scaled experiments the inability to match dimensionless quantities imposes severe limitations on its use. These limitations are particularly pronounced for metal forming processes, where a review of the literature reveals a dearth of serious significant technical works on the subject. Although numerous text books on dimensional analysis exist, few cover solid mechanics $[1,2]$ and fewer still cover forming (none in fact found). The number of articles involving both dimensional analysis and forming [3,4] is very limited. Similarity solutions simply do not exist in practical metal forming and hence a fundamental difficulty exists when designing scaled experiments. A fundamental concern of any scaling process is whether the results obtained can be guaranteed to be representative of the associated real physics. Does the physics involved scale? The short answer is no, but despite this limitation, physical models can still have a key role to play.

The presented work introduces a novel scaling methodology which is based on scaling of the integral (transport) form of the conservation laws [5]. Unlike differential representations of the conservation laws, which are point-based, the transport equations are applied to a finite space and this feature facilitates a direct map between two control volumes in the full and scaled models. In other words, using transport equations permits the direct scaling of the full-scale model. This direct scaling can be achieved by the use of Nanson's identities and time scaling to represent the physics of the full scale process at the smaller scale or vice versa.

\section{Preliminary concepts}

The presented scaling theory is based on the generalised integral form of the conservation equations [5], which can be represented mathematically as

$$
\begin{aligned}
& \frac{D^{*}}{D^{*} t} \int_{\Omega_{p s}} \rho_{p s} \psi_{p s} d V_{p s}+\int_{\Gamma_{p s}} \rho_{p s} \psi_{p s}\left(\underline{\mathrm{v}}_{p s}-\underline{\mathrm{v}}_{p s}^{*}\right) \cdot \underline{n}_{p s} d \Gamma_{p s} \\
& =-\int_{\Gamma_{p s}} \underline{J}_{p s}^{\psi} \cdot \underline{n}_{p s} d \Gamma_{p s}+\int_{\Omega_{p s}} \rho_{p s} b_{p s}^{\psi} d V_{p s}
\end{aligned}
$$

where $\rho, \psi, \underline{\mathrm{v}}, \underline{J}, b, \underline{n}$ are respectively density, physical field, velocity field, flux, source, unit normal to the boundary $\Gamma$ of the control volume $\Omega$. The subscript $p s$ in equation (1) denotes the variable associated with the physical space (unscaled model) and the star derivative $D^{*} / D^{*} t\left(=\partial /\left.\partial t\right|_{\mathbf{x}^{*}}\right)$ is temporal derivative corresponding to the map $\mathbf{x}\left(\mathbf{X}^{*}, t\right): \Omega_{P S}^{*} \mapsto \Omega_{p s}$ between the reference space $\Omega_{P S}^{*}$ and the moving control volume $\Omega_{p s}$ in the physical space [5-6]. This star-time derivative plays a similar rule as the well-known material derivative and gives rise to the definition of the control volume velocity $\underline{\mathrm{v}}_{p s}^{*}=D^{*} \mathbf{x} / D^{*} t$.

\footnotetext{
${ }^{\mathrm{a}}$ Corresponding author: keith.davey@manchester.ac.uk
} 
A similar diffeomorphism map can be considered for the trial-scaled space, i.e. $\mathbf{s}\left(\mathbf{S}^{*}, \tau\right): \Omega_{T S}^{*} \mapsto \Omega_{t s}$ which gives the velocity of $\Omega_{t s}$ as $\underline{\mathrm{v}}_{t s}^{*}=D^{*} \mathrm{~s} / D^{*} \tau$, where $D^{*} / D^{*} \tau \equiv \partial /\left.\partial \tau\right|_{\mathbf{s}^{*}}$. The symbol used for time in this map $(\tau)$ is purposely different from the physical-space map symbol $(t)$, which indicates that time in the trial space can run at a different rate from that in the physical space. Based on this newly defined diffeomorphism the transport equation for the trial space is

$$
\begin{aligned}
& \frac{D^{*}}{D^{*} \tau} \int_{\Omega_{t s}} \rho_{t s} \psi_{t s} d V_{t s}+\int_{\Gamma_{t s}} \rho_{t s} \psi_{t s}\left(\underline{\mathrm{v}}_{t s}-\underline{\mathrm{v}}_{t s}^{*}\right) \cdot \underline{n}_{t s} d \Gamma_{t s} \\
& =-\int_{\Gamma_{t s}} \underline{J}_{t s}^{\psi} \cdot \underline{n}_{t s} d \Gamma_{t s}+\int_{\Omega_{t s}} \rho_{t s} b_{t s}^{\psi} d V_{t s}
\end{aligned}
$$

Although presently there is no obvious relationship between equations (1) and (2), the transport phenomena in the physical and trial spaces can be related by assuming the existence of a smooth map $\mathbf{x}(\mathbf{s}, \tau)$ such that

$$
d x_{i}=\frac{\partial x_{i}}{\partial s_{j}} d s_{j}+\left.\frac{\partial x_{i}}{\partial \tau}\right|_{\mathbf{s}} d \tau
$$

In addition the time variables in the physical and trial spaces can be related by

$$
d t=h(\tau) d \tau
$$

where $h$ is a bijection map.

It is convenient here to assume $\mathbf{x}$ to be a function of $\mathbf{s}$ only; then equation (3) can be reduced to $\mathbf{d x}=F_{s} \cdot \mathbf{d s}$, where $F_{s}=\partial \mathbf{x} / \partial \mathbf{s}$. This deformation tensor gives rise to Nanson's geometric identities $d V_{p s}=\left|F_{s}\right| d V_{t s}$ and $\mathbf{d} \Gamma_{p s}=\left|F_{s}\right| \mathbf{d} \Gamma_{t s} \cdot F_{s}^{-1}, \quad$ where $\quad \mathbf{d} \Gamma_{p s}=\underline{n}_{p s} \mathrm{~d} \Gamma_{p s} \quad$ and $\mathbf{d} \Gamma_{t s}=\underline{n}_{t s} \mathrm{~d} \Gamma_{t s}$. By substituting these relationships into equation (1), it can rewritten as

$$
\begin{aligned}
& \frac{1}{h(\tau)} \frac{D^{*}}{D^{*} \tau} \int_{\Omega_{s s}^{*}} \rho_{p s} \psi_{p s}\left|F_{s}\right| d V_{t s}+ \\
& \int_{\Gamma_{t s}^{*}} \rho_{p s} \psi_{p s}\left|F_{s}\right| F_{s}^{-1} \cdot\left(\underline{\mathrm{v}}_{p s}-\underline{\mathrm{v}}_{p s}^{*}\right) \cdot \underline{n}_{t s} d \Gamma_{t s}= \\
& -\int_{\Gamma_{t s}^{*}}\left|F_{s}\right| F_{s}^{-1} \cdot \underline{J}_{p s}^{\psi} \cdot \underline{n}_{t s} d \Gamma_{t s}+\int_{\Omega_{t s}^{*}} \rho_{p s} b_{p s}^{\psi}\left|F_{s}\right| d V_{t s}
\end{aligned}
$$

Equations (2) and (5) are identical if

$$
\begin{gathered}
\rho_{t s} \psi_{t s}=\alpha^{\psi} \rho_{p s} \psi_{p s}\left|F_{s}\right| \\
\underline{\mathrm{v}}_{t s}-\underline{\mathrm{v}}_{t s}^{*}=h F_{s}^{-1} \cdot\left(\underline{\mathrm{v}}_{p s}-\underline{\mathrm{v}}_{p s}^{*}\right) \\
\underline{J}_{t s}^{\psi}=\alpha^{\psi} h\left|F_{s}\right| F_{s}^{-1} \cdot \underline{J}_{p s}^{\psi}
\end{gathered}
$$

$$
\rho_{t s}^{\psi} b_{t s}=\alpha^{\psi} h \rho_{p s} b_{p s}^{\psi}\left|F_{s}\right|
$$

where the scalar $\alpha^{\psi}$ is involved for slightly greater generality. Decoupling of control volume and material velocities is achieved on setting $\underline{\mathrm{v}}_{t s}^{*}=h F_{s}^{-1} \cdot \underline{\mathrm{v}}_{p s}^{*}$ and $\underline{\mathrm{v}}_{t s}=h F_{s}^{-1} \cdot \underline{\mathrm{v}}_{p s}$, which is sufficient for Equation (6b).

\section{Isotropic scaling}

The theoretical and practical aspects of isotropic scaling are presented in this section. In isotropic similarity the physical parameters are scaled with the same ratio in all principal directions. This condition simplifies the secondorder tensor $F_{s}$ to $\beta I$ where $\beta$ is scalar scaling factor and $I$ is identity tensor. Substitution of $\left|F_{s}\right|=\beta^{3}$ into equations $(6 \mathrm{a}-6 \mathrm{~d})$ results

$$
\begin{gathered}
\rho_{t s} \psi_{t s}=\alpha^{\psi} \beta^{3} \rho_{p s} \psi_{p s} \\
\underline{\mathrm{v}}_{t s}^{*}=h \beta^{-1} \underline{\mathrm{v}}_{p s}^{*} \\
\underline{\mathrm{v}}_{t s}=h \beta^{-1} \underline{\mathrm{v}}_{p s} \\
\underline{J}_{t s}^{\psi}=\alpha^{\psi} h \beta^{2} \underline{J}_{p s}^{\psi} \\
\rho_{t s} b_{t s}^{\psi}=\alpha^{\psi} h \beta^{3} \rho_{p s} b_{p s}^{\psi}
\end{gathered}
$$

It is evident that there exists a set of physical variables $\rho_{t s}, \psi_{t s}, \underline{\mathrm{v}}_{t s}^{*}, \underline{\mathrm{v}}_{t s}, \underline{J}_{t s}^{\psi}$ and $b_{t s}^{\psi}$ along with scaling parameters $\alpha^{\psi}$ and $h$ which enables equations (1) and (2) to match. But the challenge is how to practically match the physical and trial models transport equations in the case of metal forming processes.

\subsection{Practical aspects}

The transport conservation relations involved in thermomechanical metal forming are continuity, momentum, energy and entropy which for the physical space (i.e. fullscale model) can be formulated as

$$
\begin{aligned}
& \frac{D^{*}}{D^{*} t} \int_{\Omega_{p s}} \rho_{p s} d V+\int_{\Gamma_{p s}} \rho_{p s}\left(\underline{\mathrm{v}}_{p s}-\underline{\mathrm{v}}_{p s}^{*}\right) \cdot \underline{n}_{p s} d \Gamma_{p s}=0 \\
& \frac{D^{*}}{D^{*} t} \int_{\Omega_{p s}} \rho_{p s} \underline{\mathrm{v}}_{p s} d V+\int_{\Gamma_{p s}} \rho_{p s} \underline{\mathrm{v}}_{p s}\left(\underline{\mathrm{v}}_{p s}-\underline{\mathrm{v}}_{p s}^{*}\right) \cdot \underline{n}_{p s} d \Gamma \\
& =\int_{\Gamma_{p s}} \underline{\sigma}_{p s} \cdot \underline{n}_{p s} d \Gamma_{p s}+\int_{\Omega} \rho_{p s} \underline{b}_{p s} d V_{p s}
\end{aligned}
$$




$$
\begin{aligned}
& \frac{D^{*}}{D^{*} t} \int_{\Omega_{p s}} \rho_{p s} e_{p s} d V_{p s}+\int_{\Gamma_{p s}} \rho_{p s} e_{p s}\left(\underline{\mathrm{v}}_{p s}-\underline{\mathrm{v}}_{p s}^{*}\right) \cdot \underline{n}_{p s} d \Gamma_{p s} \\
& =\int_{\Gamma_{p s}} \underline{\mathrm{v}}_{p s} \cdot \underline{\sigma}_{p s} \cdot \underline{n}_{p s} d \Gamma_{p s}-\int_{\Gamma_{p s}} \underline{q}_{p s} \cdot \underline{n}_{p s} d \Gamma_{p s}+ \\
& \int_{\Omega_{p s}} \rho_{p s} Q_{p s} d V+\int_{\Omega_{p s}} \rho_{p s} \underline{\mathrm{v}}_{p s} \cdot \underline{b}_{p s} d V_{p s} \\
& \frac{D^{*}}{D^{*} t} \int_{\Omega_{p s}} \rho_{p s} s_{p s} d V_{p s}+\int_{\Gamma_{p s}} \rho_{p s} s_{p s}\left(\underline{\mathrm{v}}_{p s}-\underline{\mathrm{v}}_{p s}^{*}\right) \cdot \underline{n}_{p s} d \Gamma_{p s} \\
& =-\int_{\Gamma_{p s}} T_{p s}^{-1} \underline{q}_{p s} \cdot \underline{n}_{p s} d \Gamma_{p s}+\int_{\Omega_{p s}} \rho_{p s} T_{p s}^{-1} Q_{p s} d V_{p s}+\dot{S}_{i}
\end{aligned}
$$

where $e=u+\frac{1}{2} \underline{\mathrm{v}} \cdot \underline{\mathrm{v}}, u$ is stationary internal energy, and $s$ is specific entropy, $\underline{q} \cdot \underline{n}$ is heat flux, $Q$ represents a heat source, $\underset{\sigma}{\sigma}$ is the Cauchy stress tensor, $\underline{b}$ is a body force and $\dot{S}_{i} \geq 0$ is associated with irreversibility.

\subsubsection{Scaling of Mass}

For the conservation of mass or continuity transport equation $\psi=1, \underline{J}=\underline{0}$ and $b=0$ where for similarity system of equations (7a-e) give $\rho_{t s}=\alpha^{\rho} \beta^{3} \rho_{p s}$, $\underline{\mathrm{v}}_{t s}^{*}=h \beta^{-1} \underline{\mathrm{v}}_{p s}^{*}, \underline{\mathrm{v}}_{t s}=h \beta^{-1} \underline{\mathrm{v}}_{p s}, \underline{J}_{t s}^{\psi}=\underline{0}$ and $\rho_{t s} b_{t s}^{\psi}=0$. The relationship of principal interest here is

$$
\rho_{t s}=\alpha^{\rho} \beta^{3} \rho_{p s}
$$

For example for steel-aluminium case where the physical model material is steel and the trial model material is aluminium, the density scaling factor $\alpha^{\rho}$ is $\left(1 / \beta^{3}\right)\left(\rho_{\text {aluminium }} / \rho_{\text {steel }}\right)$.

\subsubsection{Scaling of Momentum}

As equation (9) indicates the physical field and the flux terms in the conservation of momentum are respectively $\underline{\mathrm{v}}$ and $\underline{\underline{\sigma}}$, which on replacing them in equations (7a-e) provides $\quad \rho_{t s} \underline{\mathrm{v}}_{t s}=\alpha^{-} \beta^{3} \rho_{p s} \underline{\mathrm{v}}_{p s}, \quad \underline{\mathrm{v}}_{t s}^{*}=h \beta^{-1} \underline{\mathrm{v}}_{p s}^{*}$, $\underline{\mathrm{v}}_{t s}=h \beta^{-1} \underline{\mathrm{v}}_{p s}, \quad \underline{\sigma}_{t s}=\alpha^{\underline{\mathrm{v}}} h \beta^{2} \underline{\underline{\sigma}}_{p s} \quad$ and $\rho_{t s} \underline{b}_{t s}=\alpha^{\underline{v}} h \beta^{3} \rho_{p s} \underline{b}_{p s}$.

Combination of relation $\rho_{t s} \underline{\mathrm{v}}_{t s}=\alpha^{-} \beta^{3} \rho_{p s} \underline{\mathrm{v}}_{p s}$ with equation (12) and the constraint $\underline{\mathrm{v}}_{t s}=h \beta^{-1} \underline{\mathrm{v}}_{p s}$ results

$$
\alpha^{\underline{\mathrm{V}}}=h \beta^{-1} \alpha^{\rho}
$$

Also the relation $\underline{\underline{\sigma}}_{t s}=\alpha^{\underline{\mathrm{v}}} h \beta^{2} \underline{\underline{\sigma}}_{p s}$ can be used in any constitutive model. For example in the case of von-Mises plasticity model, $\underset{=}{\sigma_{t s}^{\prime}}: \underline{\sigma}_{t s}^{\prime}=\frac{2}{3} \bar{\sigma}_{t s}^{2}$ and $\underset{=p s}{\sigma^{\prime}}: \underline{\sigma}_{p s}^{\prime}=\frac{2}{3} \bar{\sigma}_{p s}^{2}$ where $\bar{\sigma}$ and $\stackrel{\sigma^{\prime}}{=}$ are the effective stress and the deviatoric stress tensor such that $\bar{\sigma}_{t s}=\alpha^{\underline{v}} h \beta^{2} \bar{\sigma}_{p s}$ and $\underline{\underline{\sigma}}_{t s}^{\prime}=\alpha^{\underline{v}} h \beta^{2} \underline{\underline{\sigma}}_{p s}^{\prime}$. Using equation (13) and considering $\bar{\sigma}_{t s}=Y_{t s}$ and $\bar{\sigma}_{p s}=Y_{p s}$ results in

$$
h=\sqrt{\frac{1}{\alpha^{\rho} \beta} \frac{Y_{t s}}{Y_{p s}}}
$$

where $Y_{t s}$ and $Y_{p s}$ are the trial and physical material models yield stresses.

\subsubsection{Scaling of Energy}

In this case the physical field $\psi$, the flux $\underline{J}$ and the body force $b$ are respectively $e, \underline{\mathrm{v}} \cdot \underline{\sigma}$ and $\underline{\mathrm{v}} \cdot \underline{b}$. Adopting the same methodology by using equations (7a,d,e) provides $\quad \rho_{t s} e_{t s}=\alpha^{e} \beta^{3} \rho_{p s} e_{p s}$, $\underline{\mathrm{v}}_{t s} \cdot \underline{\sigma}_{t s}=\alpha^{\mathrm{e}} h \beta^{2} \underline{\mathrm{v}}_{p s} \cdot \underline{\sigma}_{p s}, \quad \rho_{t s} \underline{b}_{t s}=\alpha^{\underline{\mathrm{v}}} h \beta^{3} \rho_{p s} \underline{b}_{p s} . \quad$ Вy using equation (12) the first relationship can be expanded as $\alpha^{\rho}\left(u_{t s}+\frac{1}{2} \mathrm{v}_{t s}^{2}\right)=\alpha^{e}\left(u_{p s}+\frac{1}{2} \mathrm{v}_{p s}^{2}\right)$ and consequently be separated into two parts as

$$
\alpha^{\rho} u_{t s}=\alpha^{e} u_{p s}
$$

and

$$
\alpha^{\rho} \underline{\mathrm{v}}_{t s} \cdot \underline{\mathrm{v}}_{t s}=\alpha^{e} \underline{\mathrm{v}}_{p s} \cdot \underline{\mathrm{v}}_{p s}
$$

where the last relationship together with $\alpha^{\rho} \underline{\mathrm{v}}_{t s}=\alpha^{\underline{\mathrm{v}}} \underline{\mathrm{v}}_{p s}$, obtained from the scaling of momentum, gives

$$
\alpha^{\rho} \alpha^{e}=\alpha^{-\underline{\mathrm{v}}} \alpha^{\underline{\mathrm{v}}}
$$

Also applying the similarity relations to the heat flux and heat-source terms give

$$
\underline{q}_{t s}=\alpha^{e} h \beta^{2} \underline{q}_{p s}
$$

$$
\rho_{t s} Q_{t s}=\alpha^{e} h \beta^{3} \rho_{p s} Q_{p s}
$$

Based on equation (17), the other two energy similarity relations, $\underline{\mathrm{v}}_{t s} \cdot \underline{\underline{\sigma}}_{t s}=\alpha^{\mathrm{e}} h \beta^{2} \underline{\mathrm{v}}_{p s} \cdot \underline{\underline{\sigma}}_{p s} \quad$ and 
$\rho_{t s} \underline{\mathrm{v}}_{t s} \cdot \underline{b}_{t s}=\alpha^{e} h \beta^{3} \rho_{p s} \underline{\mathrm{v}}_{p s} \cdot \underline{b}_{p s}$, do not provide any extra information and result in the same relationships as determined by the momentum scaling, i.e. $\underline{\underline{\sigma}}_{t s}=\alpha^{\underline{\mathrm{v}}} h \beta^{2} \underline{\underline{\sigma}}_{p s}$ and $\rho_{t s} \underline{b}_{t s}=\alpha^{\underline{\mathrm{v}}} h \beta^{3} \rho_{p s} \underline{b}_{p s}$.

\subsubsection{Scaling of Entropy}

Applying similarity equations (7a-e) to the entropy equation results in 6 relationships. Only two of them are not duplications of the relationships determined on scaling of the energy and momentum equations. These relationships are $\rho_{t s} s_{t s}=\alpha^{s} \beta^{3} \rho_{p s} s_{p s}$ or equivalently $\alpha^{\rho} s_{t s}=\alpha^{s} s_{p s}$ and the entropy-transfer similarity relationship provides

$$
\frac{\underline{q}_{t s}}{T_{t s}}=\alpha^{s} h \beta^{2} \frac{\underline{q} p s}{T_{p s}}
$$

but note that equation (18) is $\underline{q}_{t s}=\alpha^{e} h \beta^{2} \underline{q}_{p s}$, which on substitution reveals

$$
\frac{\alpha^{e}}{T_{t s}}=\frac{\alpha^{s}}{T_{p s}}
$$

which provides a proportional relationship between temperatures.

\subsection{Scaling of constitutive laws}

The scaling details presented in the previous section are only for the conservation laws but in practice experimentalists need to consider the scaling of any associated material-constitutive relationships as well. In the presented methodology, the scaling of the constitutive relations is determined by the scaling applied to the conservation equations. For example, in metal forming, stress is typically a function of strain, strain rate and temperature. For this case a Johnson-Cook material model is a common choice, which for the physicalmaterial model can be written as

$$
\begin{aligned}
& \bar{\sigma}_{p s}= \\
& \left(Y_{p s}+B_{p s} \bar{\varepsilon}_{p s}^{n}\right)\left(1+C \ln \left(\frac{\dot{\bar{\varepsilon}}_{p s}}{\dot{\bar{\varepsilon}}_{p s}^{0}}\right)\right)\left(1-\left(\frac{T_{p s}-T_{p s}^{0}}{T_{p s}^{m e l t}-T_{p s}^{0}}\right)^{m}\right)
\end{aligned}
$$

where $Y_{p s}$ is the initial yield stress, $\dot{\bar{\varepsilon}}_{p s}^{0}$ is a reference strain rate, $T_{p s}^{0}$ is a reference temperature, $T_{p s}^{m e l t}$ is the solidus temperature and; $B, n, C$ and $m$ are coefficients determined by experimental means.

In view of the identity $\bar{\sigma}_{t s}=\alpha^{\rho} h^{2} \beta \bar{\sigma}_{p s}$, established in section 3.1.2 and the proportional relationships for temperature and strain rate with strain being dimensionless, equation (22) transforms into

$$
\begin{aligned}
& \bar{\sigma}_{t s}= \\
& \left(Y_{t s}+B_{t s} \bar{\varepsilon}_{t s}^{n}\right)\left(1+C \ln \left(\frac{\dot{\bar{\varepsilon}}_{t s}}{\dot{\bar{\varepsilon}}_{t s}^{0}}\right)\right)\left(1-\left(\frac{T_{t s}-T_{t s}^{0}}{T_{t s}^{m e l t}-T_{t s}^{0}}\right)^{m}\right)
\end{aligned}
$$

where $Y_{t s}=\alpha^{\rho} h^{2} \beta Y_{p s}, B_{t s}=\alpha^{\rho} h^{2} \beta B_{p s}$ and $\bar{\varepsilon}_{t s}=\bar{\varepsilon}_{p s}$.

Another material property of interest to thermomechanical analysis is thermal conductivity and with application of a classical Fourier law $\underline{q}_{p s}=-k_{p s} \nabla_{t s} T_{p s}$ but for similarity $\underline{q}_{t s}=\alpha^{e} h \beta^{2} \underline{q}_{p s}$ and $\nabla_{t s}=\beta \nabla_{p s}$, and since $\alpha^{s} T_{t s}=\alpha^{e} T_{p s}$, it follows that

$$
k_{t s}=\alpha^{s} h \beta k_{p s}
$$

which may or may not match the behaviour of a real material.

\subsection{Practical constraints}

According to the results of section 3.1, there are 6 scaling parameters which are $\beta, h, \alpha^{\rho}, \alpha^{\mathbf{v}}, \alpha^{e}$ and $\alpha^{s}$. But based on equations (13) and (17), $\alpha^{\underline{\mathbf{v}}}$ and $\alpha^{e}$ can be expressed in terms of other scaling factors and therefore there are only 4 independent factors. The factor $\beta$ is usually given, which means an experimentalist has 3 degrees of freedom to be quantified, i.e. $h, \alpha^{\rho}$ and $\alpha^{s}$.

In specific cases where the number of model-material properties and constitutive coefficients are less than or equal to 3 , then there can be a perfect match for the trial material model. An example might be pure-mechanical analysis of a rigid, perfectly-plastic material, where the unknowns are only $\rho_{t s}$ and $Y_{t s}$. But in general metal forming problems there are typically more than 3 unknowns for the trial-material model and therefore the material properties determined for the scaled model cannot be matched with a real material model. In other words, the calculated material model for the scaled experiment does not exist in reality. However, the experimentalist can attempt to select real-material models which approximate the unscaled model (see the case studies present in section 4).

As mentioned above there are 3 free scaling factors which their values can be chosen by an experimentalist. One approach therefore is to identify parameters of the scaled-material model that can be fixed. Deciding which material parameters should be chosen depends on the nature of the process. For example in the case studies presented in the next section $\rho_{t s}, Y_{t s}$ and $T_{t s}$ are chosen to be fixed, i.e. their values set equal to the density, yield stress for aluminium 7039 and room temperature. Fixing these 3 values results in a material model which does not exist in reality (i.e. its other material parameters do not 
match aluminium 7039 values) despite its density and yield stress being equal to those of aluminium 7039 . Therefore direct scaling does not necessarily result in a real material model for the scaled experiment. The steps of the direct scaling can be summarised as

Step 1. Selection of a trial material model for the scaled experiment (e.g. aluminium 7039).

Step 2. Fix 3 scaled model physical parameters depending on their importance for the process (e.g. yield stress, density and temperature).

Step 3. Calculation of scaling parameters, i.e. $h, \alpha^{\rho}$, $\alpha^{\underline{\mathbf{v}}}, \alpha^{e}$ and $\alpha^{s}$.

Step 4. Calculation of the material properties and constitutive relation coefficient for the scaled model (e.g. coefficients of the Johnson-Cook plasticity model).

Applying equations (7a-e) to evaluate $p s$ variables using $t s$ values is called reverse scaling. It is evident that applying the reverse scaling to the scaled material model calculated in step 4 gives exactly the same material model as the full-scale physical model. On the other hand, applying the reverse scaling to the real trial material model (e.g. aluminium 7039) does not match the unscaled material model but it provides an approximation for the full-scale model behaviour.

\section{Case studies}

In this section hot forging of a cylinder is considered as the case study. The dimensions of the unscaled physical model are radius $\left(r_{p s}\right)=15 \mathrm{~mm}$ and length $\left(l_{p s}\right)=30 \mathrm{~mm}$ and it is made of steel 4340 . The dimensional scaling factor $\beta$ is set to be 2 and therefore the dimensions of the scaled trial model are $r_{t s}=7.5 \mathrm{~mm}$ and $l_{t s}=15 \mathrm{~mm}$. The cylinder is placed between two rigid plates and deformed in the axial direction by half of its length (see Figure 1). The finite element analyses are performed using ABAQUS software [7]. The consistent system of units used are $\mathrm{kg}, \mathrm{mm}, \mathrm{ms}$, $K, k N, G P a$ and $J$ respectively for the mass, length, time, temperature, force, stress and energy.

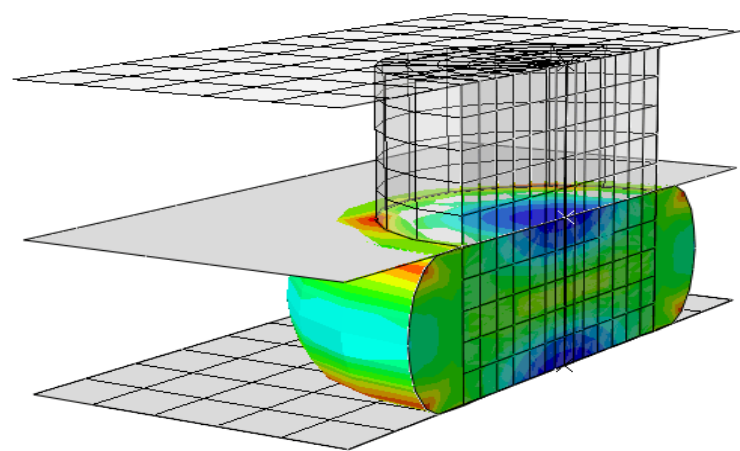

Figure 1. Un-deformed/deformed full-scale (half) steel model

The process is thermo-mechanical and consists of two steps:
Step 1. Ensure the temperature of the full-scale model reaches $700 \mathrm{~K}$ (scaled model to run at the room temperature $293 \mathrm{~K}$ ).

Step 2. Applying axial displacement by amount of $l_{p s} / 2$ for unscaled model and $l_{t s} / 2$ for the scaled model.

The natural heat convection between the surface of the cylinder and the surrounding area (dry air) is modelled during step 2 . The time process of the unscaled model for step 1 is $0.01 \mathrm{~ms}$ and for step 2 is $1 \mathrm{~ms}$, i.e. an high-loading rate typical to impact forming is considered. And the time processes of the scaled model depend on the selected trial material, i.e. time scaling factor $h$. Two possibilities are considered for the trial materials in the next subsections. In the first trial the behaviour of aluminium is adopted as the trial material model and its action under reverse scaling is studied. A tungsten alloy is considered as the second trial material in section 4.2.

\subsection{Direct/Reverse scaling I: steel-aluminium}

For the first trial, aluminium 7039 is used as the scaled experiment material. The thermo-mechanical material properties and Johnson-Cook relation coefficients for the full-scale model (steel 4340) and the scaled model (Al 7039) are presented in Table 1 . The symbols c, $\alpha_{\text {exp }}, h_{\text {conv }}$ and $\mu$ represent respectively the specific heat, thermal expansion, convection film coefficient and friction coefficients. The values of the variables are given in consistent system of units $(\mathrm{kg}, \mathrm{mm}, \mathrm{ms}, \mathrm{K})$.

Table 1. Material constants for scaled and unscaled models [8].

\begin{tabular}{|c|c|c|c|}
\hline 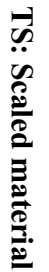 & 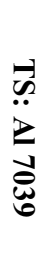 & 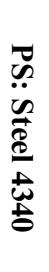 & 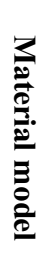 \\
\hline 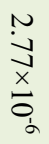 & $\begin{array}{l}\stackrel{N}{v} \\
\text { x } \\
\stackrel{x}{\sigma} \\
\dot{\alpha}\end{array}$ & $\begin{array}{l}\underset{\infty}{\infty} \\
\omega \\
\times \\
\overrightarrow{0} \\
\alpha\end{array}$ & $\nabla$ \\
\hline$\stackrel{\infty}{\cup^{\prime}}$ & Ј & 용 & 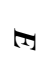 \\
\hline & 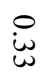 & i & 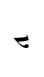 \\
\hline$\underset{\text { w }}{\omega}$ & $\begin{array}{l}\text { w } \\
\text { w }\end{array}$ & $\begin{array}{l}\stackrel{0}{v} \\
\text { ơ }\end{array}$ & $\checkmark$ \\
\hline$\stackrel{0}{\text { i }}$ & $\underset{\perp}{\stackrel{\leftrightarrow}{\infty}}$ & in & $\infty$ \\
\hline$\stackrel{0}{\text { i }}$ & $\stackrel{\circ}{\underline{N}}$ & $\stackrel{\circ}{\circ}$ & $m_{0}$ \\
\hline
\end{tabular}




\begin{tabular}{|c|c|c|c|}
\hline$\stackrel{8}{\circ}$ & $\stackrel{\circ}{\circ}$ & $\stackrel{\circ}{\stackrel{\circ}{\not}}$ & 0 \\
\hline$\stackrel{P}{ \pm}$ & $\stackrel{\ominus}{ \pm}$ & i் & $=$ \\
\hline- & - & $\dot{\tilde{c}}$ & $\Xi$ \\
\hline પ્ઠ & $\stackrel{\infty}{\cup}$ & ప్ర & 츤 \\
\hline 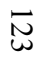 & స్త & 记 & to \\
\hline 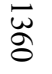 & $\begin{array}{l}\infty \\
\infty\end{array}$ & 出 & 0 \\
\hline 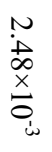 & $\begin{array}{l}\overrightarrow{\vec{a}} \\
\vec{x} \\
\overrightarrow{0} \\
\vec{i}\end{array}$ & 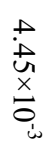 & $\nabla$ \\
\hline 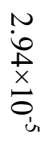 & 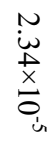 & 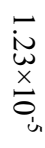 & 8 \\
\hline 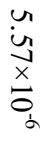 & $\begin{array}{l}u \\
\ddot{u} \\
\vec{x} \\
0 \\
0 \\
\dot{\alpha}\end{array}$ & $\begin{array}{l}\frac{\tilde{x}}{0} \\
{ }_{\alpha}\end{array}$ & 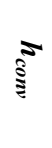 \\
\hline ஜூ & ஜू & : & $=$ \\
\hline
\end{tabular}

As it is highlighted in Table 1, the density and yield stress of the scaled material is set to be equal to the trial material model (Al 7039). This is due to the two degrees of freedom provided by free scaling factors $h$ and $\alpha^{\rho}$. Since there are in total 3 free scaling factors $\left(h, \alpha^{\rho}\right.$ and $\alpha^{s}$ ), the scaling factor $\alpha^{s}$ is still free and it allows to fix another physical parameter of the scaled experiments. In this case study the applied temperature to the scaled cylinder is fixed to be the room temperature $(293 \mathrm{~K})$ while the applied temperature to the full-scale model is $700 \mathrm{~K}$. The other two scaling factors $\alpha^{\underline{\mathbf{v}}}$ and $\alpha^{e}$ can be calculated using the values of the free scaling factors $h$, $\alpha^{\rho}$ and $\alpha^{s}$ through equations (13) and (17). The scaling parameters values for steel-aluminium combination are presented in Table 2.

Table 2. Scaling factors for steel-aluminium combination.

\begin{tabular}{|c|c|c|c|c|c|c|}
\hline Factor & $\beta$ & $h$ & $\alpha^{\rho}$ & $\alpha^{\mathrm{v}}$ & $\alpha^{e}$ & $\alpha^{s}$ \\
\hline Value & 2 & 2.19 & $\begin{array}{c}4.42 \\
\times 10^{-2}\end{array}$ & $\begin{array}{c}4.85 \\
\times 10^{-2}\end{array}$ & $\begin{array}{c}5.32 \\
\times 10^{-2}\end{array}$ & $\begin{array}{c}1.27 \\
\times 10^{-1}\end{array}$ \\
\hline
\end{tabular}

The process time of the unscaled model for the step 2 is $1 \mathrm{~ms}$ and for the scaled model is $0.455 \mathrm{~ms}$, i.e. $1 / \mathrm{h}$. It is clear from Table 1 data that the scaled material model does not exist in reality but the selected trial material ( $\mathrm{Al}$ 7039) provides a first attempt at the scaling experiment. The finite element results for the axial force-displacement of the scaled and trial material models are depicted in Figure 2. The erratic behaviour at the initial stages of the results is due to the thermal step (i.e. step 1).

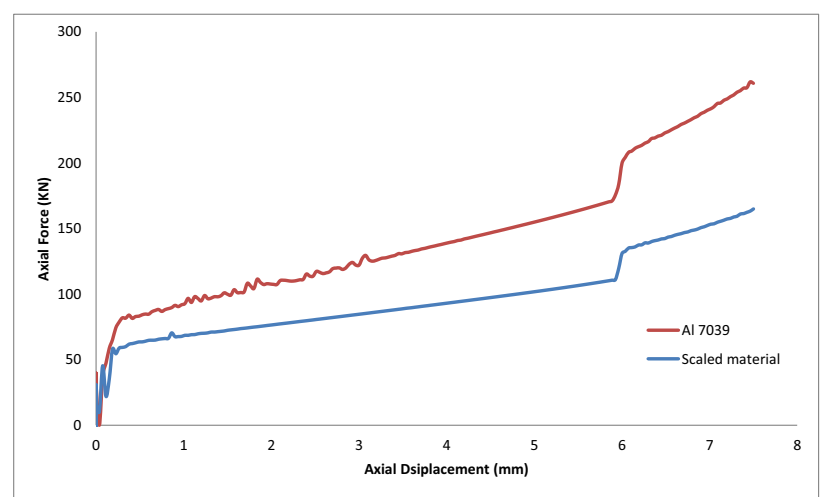

Figure 2. Force-displacement plots for scaled and trial material models

Applying the reverse scaling (see section 3.3) to the scaled material presented in Table 1 gives exactly the same material properties as the unscaled material model (steel 4340) while the reverse map of the Al 7039 material model results in a different material model with the following constants: $\rho=7.83 \times 10^{-6}, E=164.5, v=0.33$, $Y=0.792, \quad B=0.80, \quad \dot{\varepsilon}^{0}=0.1, \quad C=0.014, \quad n=0.41, \quad m=1$, $T^{\text {nelt }}=2095.2, \quad T^{0}=700, \quad c=306.24, \quad K=2.51 \times 10^{-2}$, $\alpha_{\text {exp }}=9.79 \times 10^{-6}, h_{\text {conv }}=5 \times 10^{-6}$, and $\mu=0.6$. These values are worked out in the consistent system of units $(\mathrm{kg}, \mathrm{mm}$, $m s, K)$. The axial force-displacement behaviour of the physical model (steel 4340), reversed scaled material and reversed Al 7039 models under the original (full-scale) loading conditions are compared in Figure 3.

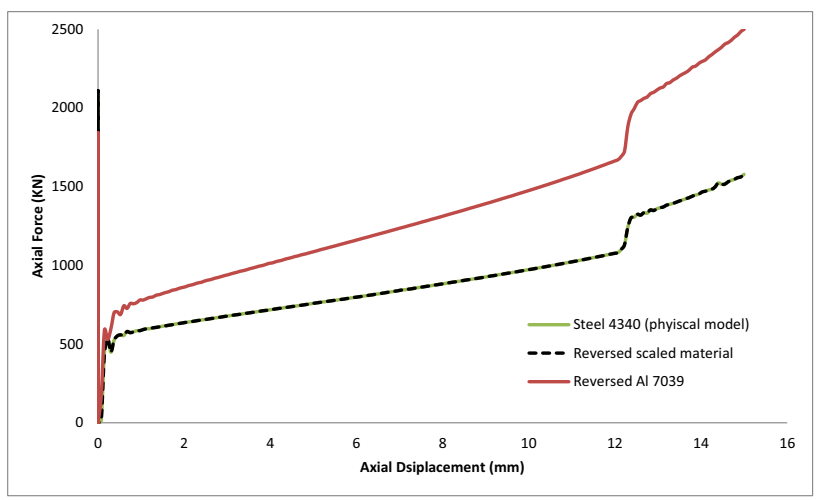

Figure 3. Force-displacement plots for physical and reversed material models.

The results show that the selection of $\mathrm{Al} 7039$ as the trial material model gives rises to significantly higher loads than what is required in the design of the scaling experiment. In next subsection, another trial material (tungsten alloy) is used to provide a closer approximation for the thermo-mechanical behaviour of the full-scale physical model. 


\subsection{Direct/Reverse scaling II: steel-tungsten}

The geometry, dimensions and thermo-mechanical loadings of this case study are exactly similar to the previous case study. The material of the full scale physical model is the same (i.e. steel 4340) but the trial material model is selected to be tungsten alloy $(0.07 \mathrm{Ni}$, $0.03 \mathrm{Fe})$. The material properties and Johnson-Cook coefficients for the selected tungsten alloy are: $\rho=1.70 \times 10^{-5}, E=450, v=0.28, Y=1.506, B=0.177, \varepsilon^{\circ}=0.1$, $C=0.016, n=0.12, \quad m=1, T^{\text {melt }}=1723, T^{0}=293, c=134$, $K=1 \times 10^{-2}, \alpha_{\exp }=5 \times 10^{-6}, h_{\text {conv }}=5 \times 10^{-6}$, and $\mu=0.6$ [8]. The calculated scaling factors values for the steeltungsten scaling experiment are presented in Table 3.

Table 3. Scaling factors for steel-tungsten combination.

\begin{tabular}{|c|c|c|c|c|c|c|}
\hline Factor & $\beta$ & $h$ & $\alpha^{\rho}$ & $\alpha^{\mathrm{v}}$ & $\alpha^{e}$ & $\alpha^{s}$ \\
\hline Value & 2 & 1.87 & $\begin{array}{c}2.71 \\
\times 10^{-1}\end{array}$ & $\begin{array}{l}2.54 \\
\times 10^{-1}\end{array}$ & $\begin{array}{c}2.38 \\
\times 10^{-1}\end{array}$ & $\begin{array}{c}5.68 \\
\times 10^{-1}\end{array}$ \\
\hline
\end{tabular}

By adopting the same approach used for the previous case study the scaled material constants can be worked out as follow: $\rho=1.70 \times 10^{-5}, E=380, v=0.3, Y=1.506$, $B=0.97, \dot{\varepsilon}^{0}=0.18, C=0.014, n=0.26, m=1.03, T^{\text {melt }}=750$, $T^{0}=123, \quad c=994, \quad K=9.46 \times 10^{-3}, \quad \alpha_{\exp }=2.94 \times 10^{-5}$, $h_{\text {conv }}=2.13 \times 10^{-5}$, and $\mu=0.6$. The process time of the unscaled model for the step 2 is $1 \mathrm{~ms}$ and for the scaled model is $0.534 \mathrm{~ms}$, i.e. $1 / \mathrm{h}$. The axial force-displacement behaviour of the scaled models (i.e. trial and scaled materials) is depicted in Figure 4.

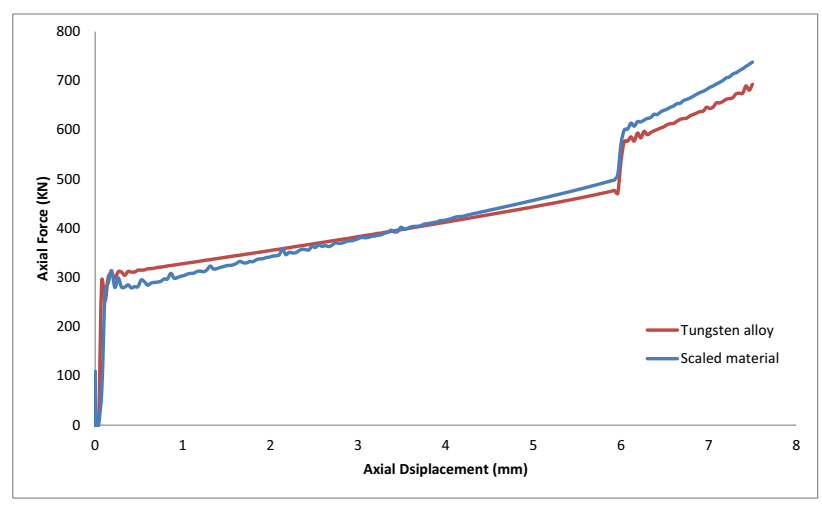

Figure 4. Force-displacement plots for scaled and trial materials models

Applying the reverse scaling (see section 3.3) to the scaled material gives exactly the same material properties as the full-scale model material (steel 4340) while the reverse map of the trial material (tungsten alloy) results in a different material model with the following constants: $\rho=7.83 \times 10^{-6}, E=236.65, \quad v=0.28, \quad Y=0.792$, $B=0.093, \dot{\varepsilon}^{0}=0.1, C=0.016, n=0.12, m=1, T^{m e l t}=4116.3$, $T^{0}=700, \quad c=64.04, \quad K=4.70 \times 10^{-3}, \quad \alpha_{\exp }=2.09 \times 10^{-6}$, $h_{\text {conv }}=5 \times 10^{-6}$, and $\mu=0.6$. The axial force-displacement behaviour of the physical model (steel 4340), reversed scaled material and reversed tungsten alloy models are shown in Figure 5.

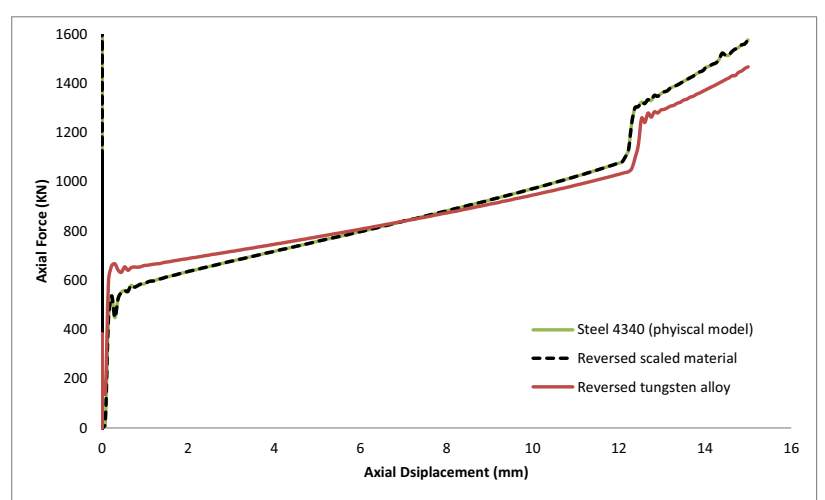

Figure 5. Force-displacement plots for physical and reversed material models.

These results indicate that tungsten alloy $(0.07 \mathrm{Ni}$, $0.03 \mathrm{Fe}$ ) as the trial material model provides a close match for the scaling of this forming process. The results provide confidence for adopting a tungsten-alloy scaled model to represent the physics of the steel billet.

\section{Conclusions}

The paper is concerned with a novel methodology in isotropic scaling of thermo-mechanical processes and the following conclusions can be drawn from the presented work:

- The generalised integral form of the conservation laws (i.e. the transport approach) can be scaled and used to represent continuum physics on a scaled space.

- The presented theory provides a general framework for both isotropic and anisotropic scaling although its application in this paper is limited to isotropic scaling.

- The presented theory and numerical results confirm the applicability of the presented scaling methodology to thermo-mechanical processes.

\section{References}

1. L.I. Sedov, Similarity and dimensional methods in mechanics (CRC Press, $10^{\text {th }}$ Ed. 1993)

2. T. Qing-Ming, Dimensional analysis: with case studies in Mechanics (Springer, 2011)

3. J.S. Ajiboye, K.H. Jung, Y.T. Im, J. Mech. Sci. Technol. 24 115-118 (2010)

4. A.A. Ezra, F.A. Penning, Exp. Mech. 2 234-239 (1962)

5. R. Darvizeh, K. Davey, Int. J. Impact Eng. 82 59-73 (2015)

6. R. Darvizeh, K. Davey, Comput. Struct. 164 145-160 (2016)

7. ABAQUS 6.14, Dassault Systèmes (Providence, RI, USA)

8. M.A. Meyers, Dynamic behavior of materials (Wiley 1994) 